\title{
Local Binary Pattern Based Iris Recognition Model
}

\author{
Mohammed Hafeez M K., Asst. Professor, PACE, Mangalore, India, hafiz123ster@gmail.com \\ Sharmila Kumari M., Professor \& Head, PACE, Mangalore, India, sharmilabp@gmail.com
}

Abstract: Iris is a unique biometric tool, secure and reliable in recognizing an individual based on the texture information of human physiology. The Local Binary Pattern method uses descriptors based on histograms of Local Binary Pattern. In developed algorithm, Local Binary Pattern (LBP) histograms of iris images are extracted and concatenated into single enhanced histogram. It can be computed by nearest neighbor classifier and iris recognition is performed using Hamming distance as dissimilarity measure. We have conducted experimentation on CASIA dataset. From the experimental results, it is proved that the Robust LBP technique for iris recognition is more accurate than the conventional LBP.

Keywords - Biometrics, Casia dataset, Feature Extraction, Hamming Distance, Local Binary Pattern, Robust Local Binary pattern .

\section{INTRODUCTION}

Nowadays the most used authentication methods rely on biometric elements. Biometrics is considered to be the unique (personal) physical/logical traits of human body [1]. Any person could be identified by extracting some characteristics from different parts of the body: face, iris, periocular region, fingerprint, veins, DNA.

Iris recognition is a physiological biometric solution identifies or confirms the identity of a subject by analyzing the random texture of the iris. The texture information in the human iris is formed by 10 months of age, and remains unique and unchanged throughout one's lifetime [2][22]. Even genetically identical twins will be having different Iris textures [23]. On the other hand, iris recognition is a noninvasive technique, flexible and reliable, which could be easily integrated into other existing biometric systems.

It's been concluded by the researchers in the iris biometric field that there are four main stages in the iris biometrics system: image acquisition, iris region segmentation, iris texture analysis and finally the matching of iris representations [6].

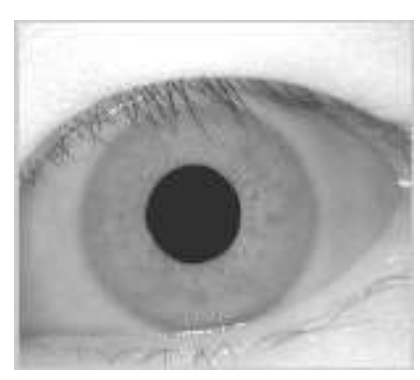

Figure 1: acquired iris image.

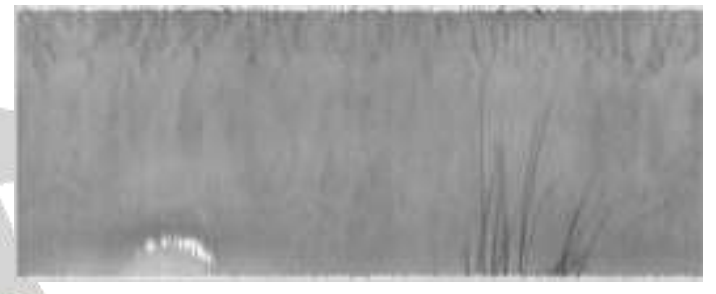

Figure 2: Unwrapped Segmented iris image.

The photo of an individual's eye is captured in acquisition stage. The preprocessing and segmentation of the obtained eye image extracts the iris region. Iris normalization obtains the rectangular representation of the iris image by fixing the dimensions of segmented iris region to allow for accurate comparisons. Feature extraction uniquely encodes the iris image to creating the template from normalized image and matches this test template with reference templates. The performance of an iris system closely depends on the precision of the iris segmentation [4]. The existing methods assume that pupil is always central to an iris. Hence both pupil and iris share a central point. This inaccurate assumptions results in wrong segmentation of an iris region. The upper and the lower parts of the outer iris boundary are generally obstructed by eyelids and eyelashes, this provides problems during segmentation. These eyelids and eyelashes act as noise which needs to be eliminated to achieve optimum segmentation results [11].

LBP is one of the most powerful descriptors employed for encoding local structures and has been successfully used for many image analysis tasks including biometrics[5]. Lately a large number of variations were designed starting from this methodology. The present approach utilizes several techniques derived from LBP for iris recognition. Local Binary Pattern (LBP) is originally used for shape and texture which is based on local features .In developed method the rectangular representation of the normalized iris area is first divided into small regions from which Local Binary Pattern (LBP) operator is computed [2]. For that operator need to 
construct the histogram and finally extract those LBP histograms and concatenated into single, spatial enhanced histogram which representing the iris image. Here Features are extracted from each regions .Using nearest neighbor classifier performing the recognition with the help of Hamming distance as dissimilarity measure. According to analysis LBP provides both in terms of speed and discrimination performance. Here recognition rate is analyzed with different databases like CASIA databases.

The remainder of this paper is organized as follows. Section II provides an overview of the Local Binary Pattern. Robust Local Binary Pattern is presented in Section III. Experimental results and discussions are reported in Section IV. Section V concludes the paper and outlines future research.

\section{LOCAL BINARY PATTERN}

The Local Binary Pattern (LBP) operator is a very effective method for texture analysis. It was firstly defined as a grayscale invariant measure, derived from a local image neighborhood. LBP describes the texture with microprimitives, called textons, and their statistical placement rules.

Here Local Binary Pattern operator works with eight neighbors of pixels, using center pixel value as threshold value. Compare neighbor pixel with center pixel [15]. If neighbor pixel has higher gray value than the threshold value then one is assigned to that pixel, otherwise it gets zero value [17]. Then concatenate these eight ones or zeroes into binary code and calculate corresponding decimal value and then replace center pixel with the decimal value as shown in figure 3 .

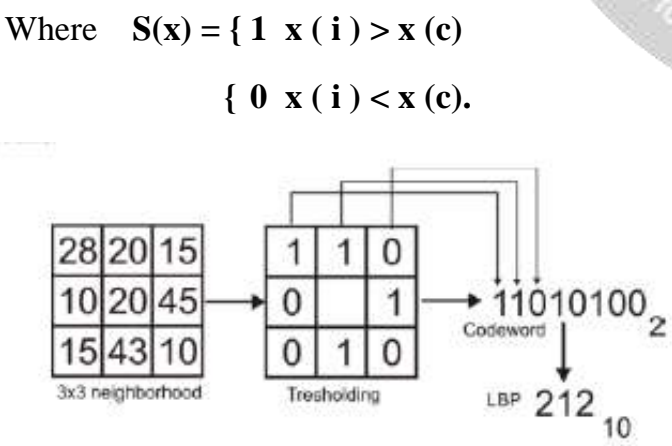

Figure 3: Local Binary Pattern.

The original 8-bit version of the LBP operator is rotation variant. To achieve multi-scale analysis and rotation invariance, the classical LBP method has been extended to arbitrary circular neighborhoods of the central pixel, known as $\mathrm{LBP}_{\mathrm{P}, \mathrm{R}}$ [14]. Figure - 4 illustrates the idea - any number of neighbors $(\mathrm{P})$ can be selected from the circular radius $(\mathrm{R})$ [12]. Neighbor samples are interpolated on the circle with equal space. If, for the basic LBP the dimensionality was 28 $=256$ local patterns, for the circular neighborhood the number increases with the number of samples.

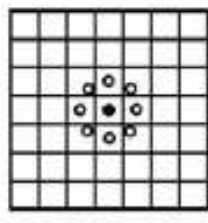

$\mathrm{P}=8, \mathrm{R}=1.0$

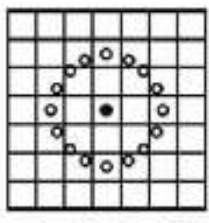

$P=16, R=2.0$

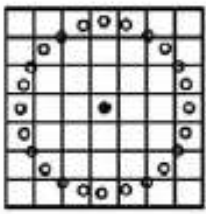

$P=24, R=3.0$
Figure 4: $L B P_{P, R}$ invariant to rotations. Neighbor sets for different $(P, R)$ values.

The $\mathrm{LBP}_{\mathrm{P}, \mathrm{R}}$ operator generates $2 \mathrm{P}$ different output values, corresponding to the $2 \mathrm{P}$ different binary patterns that can be formed by the $\mathrm{P}$ pixels in the neighbor set [10]. Ojala et al. use only the 'uniform' patterns, where the maximum number of bit-wise changes from one to zero or reverse in the circular neighborhood, is allowed to be $2[4][8]$. It was observed that uniform local patterns represent the great majority, sometimes over 90 percent of all local patterns in the image.

\section{ROBUST LOCAL BINARY PATTERN}

LBP is sensitive to noise and fails due to high intra-class variance making it unsuitable for iris recognition. The high intra-class variance is due to the discrimination of brighter image against dark background vice-versa. This problem is avoided by choosing the minimum value of the iris code obtained by comparing the original unique iris code generated using LBP and its bitwise compliment. This makes the LBP code most robust by reversing the intensities of iris features.

\section{RLBP $=\min \left(\mathbf{L B P}, \mathbf{L B P}^{-1}\right)$}

RLBP minimizes the maximum code with minimum code for example LBP compliment makes 10101100 code as 01010011 which is minimum, it makes intra-class variance minimum. RLBP is robust to noise, fluctuations and it's efficient

The object has two distinct ideas for differentiation of the object, such as Objet surface texture and object shape formed by its boundary [3]. Boundary shows higher contrast between the object and its background. Since the boundary contains the shape information that is discriminated from the surface texture for additional discriminative information.
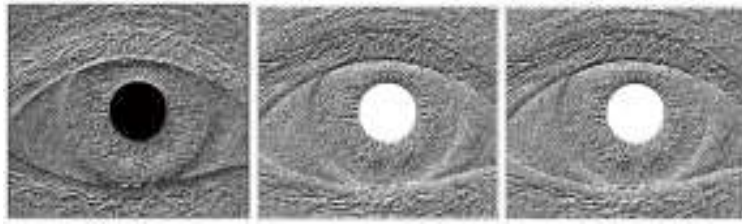

Figure 5: Processing of RLBP iris image.

Figure 5 shows the processing of the RLBP image. $1^{\text {st }}$ image shows the LBP image which then complimented shows in the $2^{\text {nd }}$ image then compared to get the final outcome of the RLBP image which is represented in the $3^{\text {rd }}$ image. 
LBP do not differentiate between a weak contrast local pattern and a strong contrast local pattern. Histogram of LBP provides frequencies of the code (i.e.) weight for each code is the same. By combining the edge and texture information into a single representation we get expected histogram.

\section{EXPERIMENTS AND PERFORMANCE ANALYSIS}

In order to evaluate the effectiveness of the above mentioned methods for the iris recognition, a publicly available CASIA iris database was used. The CASIA iris database includes 756 iris images from 108 persons ( 3 from the left eye and 4 from the right one).

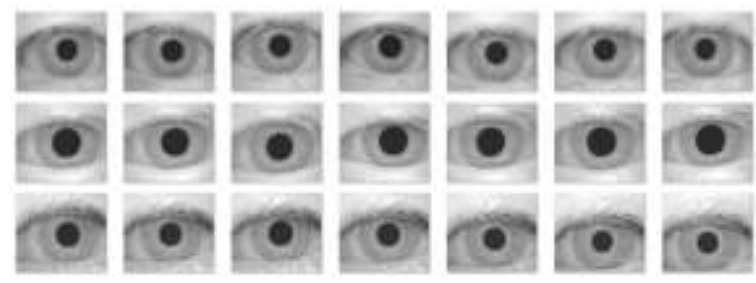

Figure 5: Samples from CASIA database.

In this chapter we carry out quantitative and qualitative comparison among the proposed techniques. The Hamming distance was used for the classification exercise. To evaluate the potential of using texture features for iris classification, the database is split into the training set and the verification set. For the experimentation purpose, the training set is obtained by splitting the CASIA database into several combination of the samples as shown in Table 1 under the Training Images and Testing Images column. The entry set $\{1,2,4,5\}$ for Training Images in Table 1 , indicates that 4 out of the 7 samples of each individual person from the CASIA dataset is considered for creating the reference templates. The remaining set $\{3,6,7\}$ for Testing Images in Table 1 are the samples taken to evaluate the performance of the system. A random iris image is selected from the Testing Image and test template is created by applying the four stages of iris recognition system. The resulting test template is compared with each reference template generated during the training stage. In order to calculate the recognition rates the training sets have been run in both LBP and RLBP. The accuracy is obtained by the error rates which are EER (Equal Error Rate), FAR (False Acceptance rate), FRR (False Rejection Rate).

\begin{tabular}{|c|c|c|c|c|}
\hline $\begin{array}{c}\text { SI. } \\
\text { No }\end{array}$ & $\begin{array}{c}\text { Training } \\
\text { Images }\end{array}$ & $\begin{array}{c}\text { Testing } \\
\text { Images }\end{array}$ & $\begin{array}{c}\text { Recognition } \\
\text { with LBP }\end{array}$ & $\begin{array}{c}\text { Recognition } \\
\text { with RLBP }\end{array}$ \\
\hline 1 & $\{1,2,4,5\}$ & $\{3,6,7\}$ & $72 \%$ & $93 \%$ \\
\hline 2 & $\{2,3,5,6\}$ & $\{1,4,7\}$ & $79 \%$ & $86 \%$ \\
\hline 3 & $\{1,3,6,7\}$ & $\{2,4,5\}$ & $83 \%$ & $89 \%$ \\
\hline 4 & $\{1,2,4,5,6\}$ & $\{3,7\}$ & $89 \%$ & $91 \%$ \\
\hline 5 & $\{2,3,5,6,7\}$ & $\{1,4\}$ & $90 \%$ & $92 \%$ \\
\hline
\end{tabular}

Table 1: Recognition accuracy of LBP and RLBP on CASIA dataset.
From Table-1 we can analyze the recognition rate for CASIA database with different number of samples for training and testing image set. Outcome of the experiment conducted is illustrated in Table 1, RLBP works better when compared to LBP.

\section{CONCLUTION}

In this paper we have discussed about local binary pattern which is the feature extraction method. To remove the drawback of the LBP we have come up with more efficient method called Robust Local Binary Pattern. The CASIA database is used for the experiments. The results shows the recognition rate is more accurate when the conventional LBP method is compared with the robust LBP method.

The table analyze the recognition rate for the CASIA database. To increase recognition rate and to reducing computing time some improvements are still possible.

\section{REFRENCES}

[1.] Vany.S, Karthi Prem.S and Udhayachandrika.A "A Robust Object Recognition using LBP, LTP and RLBP”, International Journal of Modern Trends in Engineering and Research, e-ISSN: 2349-9745, p-ISSN: 2393-8161, 2014.

[2.] T. Ojala, M. Pietikäinen, and T.Maenpaa, "Multiresolution gray-scaleand rotation invariant texture classification with local binary patterns", IEEE Trans. Pattern Analysis and Machine Intelligence, vol. 24, no. 7, pp. 971-987, 2002.

[3.] M.Y. Shams, et al. "Iris recognition based on LBP and combined LVQ classifier", International Journal of Computer Science \& Information Technology, vol. 3(5), 2011, pp. $67-78$

[4.] M Sharmila Kumari, Bheemappa Arjunappa Mudalawar, "Local Binary Pattern Based Face Recognition Model," P. A. College of Engineering, 2013.

[5.] Simina Emerich, Raul Malutan, Eugen Lupu, László Lefkovits "Patch Based Descriptors for Iris Recognition”, 978-1-5090-3899-2/16/\$31.00 (2016.

[6.] K. Royand and M. Kamel, "Multibiometric system using distance regularized level set method and particle swarm optimization," in Proc. 2012 Int. Conf. on Computer Vision and Graphics, 2012, pp. 590-599.

[7.] K. Roy and M. Kamel, "Multibiometrics system based on level set method and particle swarm optimization," in Proc. 2012 Int. Conf. on Image Analysis and Recognition, 2012, pp. 20-29.

[8.] T. Ojala, M. Pietikäinen, and T. Mäenpää, "Multire solution gray-scale and rotation invariant texture classification with local binary pattern," IEEE Trans. on 
Pattern Analysis and Machine Intelligence, vol. 24, pp. 971-987, Aug. 2002.

[9.] Z. H. Guo, D. Zhang, and D. Zhang, "A completed modeling of local binary pattern operator for texture classification," IEEE Trans. on Image Processing, vol. 19, pp. 1657-1663, May 2010.

[10.]J. Koh, V. Govindaraju, and V. Chaudhary, "A robust iris localization method using an active contour model and Hough transform," in Proc. 2010 IEEE Int. Conf. on Pattern Recog., 2010, pp. 2852-2856.

[11.]C. M. Li, C. Y. Xu, C. F. Gui, and M. D. Fox, "Distance regularized level set evolution and its application to image segmentation," IEEE Trans. Image Process, vol. 19, pp. 3243-3254, Nov. 2010.

[12.]Qichuan Tian, Hua Qu, Lanfang Zhang and Ruishan Zong," Personal Identity Recognition Approach Based on Iris Pattern", State of the art in Biometrics, Dr. Jucheng Yang (Ed.), ISBN: 978-953-307-489-4,2011.

[13.]W.W.Boles and B.Boashash,"A human identification technique using images of the iris and wavelet transform, IEEE Classification",Transactions on Signal Processing, 46(4), 1185-1188,1998.

[14.]P. Radu, K. Sirlantzis, W.G.J. Howells, F. Deravi, S. Hoque, "Information Fusion for Unconstrained Iris Recognition", International Journal of Hybrid Information Technology, Vol. 4 No. 4, October, 2011, pp. 1-12.

[15.] http://biometrics.idealtest.org/dbDetailForUser.do?id=1

[16.]Rai, Himanshu, and Anamika Yadav. "Iris recognition using combined support vector machine and Hamming distance approach." Expert Systems with Applications Vol 41, no. 2 (2014): 588-593.

[17.]Rangaswamy Y and K. B. Raja, "Straight-line Fusion based Iris Recognition using AHE, HE and DWT", Elsevier International Conference on Advanced Communication Control and Computing Technologie, pp.228-232, 2016. .

[18.]Charan S G, "Iris Recognition using feature optimization", Elsevier International conference on Applied and Theoretical Computing and Communication Technology, pp. 726-731, 2016. Vanaja Roselin E. Chirchi and L. M. Waghmare, "Feature Extraction and Pupil Detection Algorithm Used for Iris Biometric Authentication System", International Journal of Signal Processing, Image Processing and Pattern Recognition, Vol.6, No.6 (2013), pp.141-160.

[19.]U. Gawande, K. Hajari and Y. Golhar, "Novel Technique for Removing Corneal Reflection in Noisy Environment Enhancing Iris Recognition Performance", IEEE International conference on signal and information processing, pp. 1-5, 2016.

[20.]A. Satish, Adhau and D. K. Shedge, "Iris Recognition methods of a blinked eye in non-ideal Condition", IEEE International Conference on Information Processing, pp.
75-79, 2016. J. Trader, "M2SYS Blog On Biometric Technology," Delta ID, 11 June 2012. [Online]. Available: http://blog.m2sys.com/biometrichardware/ iris- recognition-vs-retina-scanning-what-are-thedifferences.

[21.]R. Dillak and M. Bintiri, "A novel approach for iris recognition", IEEE International Symposium, pp. 231236, 2016.

[22.]Rahul A Patil et al.,(2015),"Steps of Human Iris Detection for Biometric Application", International Journal of Advanced Research in Computer Engineering Vol 4,Issue 12,December 2015.

[23.]Richu Sharma and Vaneet Mohan(2016),"Biometric Identification using Iris recognition System", International Journal of Computer Application(22501797), Volume 6 - No.2,March - April 2016

[24.]Rajvir kaur and Ishpreet Singh (2016),'Enhanced Human Iris Recognition System based on Procedure of Authentication System", International Journal of Computer Applications(0975 - 8887) Volume 146 No.9.July 2016. 